\title{
Long noncoding RNA CBR3 antisense RNA 1 promotes the aggressive phenotypes of non-small-cell lung cancer by sponging microRNA-509-3p and competitively upregulating HDAC9 expression
}

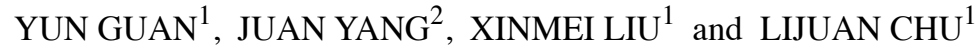 \\ Departments of ${ }^{1}$ Respiratory Medicine and ${ }^{2}$ General Surgery, \\ Weifang People's Hospital, Weifang, Shandong 261041, P.R. China
}

Received February 22, 2020; Accepted July 6, 2020

DOI: 10.3892/or.2020.7719

\begin{abstract}
Long noncoding RNA CBR3 antisense RNA 1 (CBR3-AS1) plays significant roles in the initiation and progression of osteosarcoma. The aim of the present study was to investigate the involvement of CBR3-AS1 in the development of non-small cell lung cancer (NSCLC). Reverse transcription-quantitative PCR was performed to detect CBR3-AS1 expression in NSCLC tissues and cell lines. The impacts of CBR3-AS1 on cellular proliferation, apoptosis, migration and invasiveness in vitro, and tumor growth in vivo, were investigated using the Cell Counting Kit- 8 assay, flow cytometry, Transwell migration and invasion assays, and tumor xenograft model-based analysis, respectively. The results indicated that CBR3-AS1 was markedly upregulated in NSCLC tissues and cell lines. High CBR3-AS1 expression was correlated with larger tumor size, advanced TNM stage, increased incidence of lymph node metastasis and shorter overall survival times in patients with NSCLC. Furthermore, CBR3-AS1-knockdown notably suppressed cellular proliferation, migration and invasiveness in vitro, and also promoted apoptosis and suppressed tumorigenicity in vivo. Mechanistic investigation demonstrated that CBR3-AS1 functions as a competing endogenous RNA for microRNA-509-3p (miR-509-3p) in NSCLC cells. Furthermore, miR-509-3p exerted tumor-suppressive effects in NSCLC, and histone deacetylase 9 (HDAC9) was identified as a direct target of miR-509-3p. HDAC9 expression was suppressed by CBR3-AS1 depletion, which was abolished by miR-509-3p inhibition. Further rescue experiments revealed that increasing the output of the miR-509-3p/HDAC9 axis counteracted the CBR3-AS1 depletion-induced inhibitory
\end{abstract}

Correspondence to: Professor Lijuan Chu, Department of Respiratory Medicine, Weifang People's Hospital, 151 Guangwen Road, Weifang, Shandong 261041, P.R. China

E-mail: chulijuan_001@163.com

Key words: CBR3 antisense RNA 1, microRNA-509-3p, histone deacetylase 9, non-small cell lung cancer, tumorigenesis effects on NSCLC cells. Collectively, the results of the present study indicate that the CBR3-AS1/miR-509-3p/HDAC9 pathway exerts tumor-promoting actions in NSCLC oncogenesis and progression, suggesting that this pathway is an effective target for the management of NSCLC.

\section{Introduction}

Lung cancer is not only the most commonly diagnosed cancer, but also the leading cause of cancer-associated death globally, with a predicted 2,28,150 novel cases and 1,47,510 deaths annually $(1,2)$. Non-small cell lung cancer (NSCLC) is the most prevalent pathological subtype of lung cancer and accounts for $80-85 \%$ of all lung cancer cases (3). NSCLC is classified into lung squamous cell carcinoma, lung adenocarcinoma and large-cell lung cancer, and all three pathological classifications manifest similar biological behaviors and gene mutations (4). Although vigorous attempts have been made to improve the quality of NSCLC therapy, the long-term survival of patients remains low (5). When diagnosed at an early stage, NSCLC can be effectively treated by surgical resection, chemotherapy and radiotherapy. However, most patients are diagnosed at a later or advanced stage, and are typically treated with first-line therapeutic strategies that have poor clinical efficiency (6). Therefore, advancements in diagnostic testing and therapy are necessary. An improved understanding of the mechanisms underlying NSCLC tumorigenesis may aid in the identification of promising diagnostic biomarkers and therapeutic targets.

Long noncoding RNAs (lncRNAs) belong to a large and diverse group of transcripts of $>200$ nucleotides that lack protein-coding capacity (7). In previous decades, lncRNAs have received increasing attention for their roles in numerous biological activities and diseases, particularly in human cancers (8). Differentially expressed lncRNAs have been verified in almost all human cancer types, including $\operatorname{NSCLC}(9,10)$. An increasing number of studies have shown that the dysregulation of lncRNAs plays a significant role in NSCLC tumorigenesis and progression by exerting cancer-inhibiting or oncogenic effects (11-13).

MicroRNAs (miRNAs) are a class of single-stranded noncoding RNA that can directly bind to the 3'-untranslated 
region of their target mRNAs, which triggers mRNA degradation and/or translation depression (14). Studies have shown that aberrant miRNA expression is closely associated with the initiation and development of NSCLC, and is implicated in the regulation of a wide range of tumorigenic behaviors (15-17). Multiple studies have revealed that lncRNAs are capable of interacting with miRNAs, thereby forming competing endogenous RNA (ceRNA) regulatory networks to modulate the expression and function of coding genes in NSCLC (18-20). Hence, studying the detailed roles of lncRNAs and miRNAs in NSCLC, as well as illustrating their cellular functions and interactions, may aid the diagnosis, prognostic prediction and development of therapies for NSCLC.

lncRNA CBR3 antisense RNA 1 (CBR3-AS1) has been shown to promote the initiation and progression of osteosarcoma (21). The present study aimed to explore whether CBR3-AS1 is involved in the development of NSCLC. CBR3-AS1 expression was detected in NSCLC tissues and cells lines to reveal its prognostic significance in NSCLC, and to determine its influence on aggressive phenotypes of NSCLC cells. The study also aimed to elucidate the potential molecular mechanisms underlying the oncogenic roles of CBR3-AS1, which may provide potential targets for novel diagnostic and therapeutic strategies.

\section{Materials and methods}

Patients and tissue samples. In total, 57 pairs of tumor and adjacent healthy tissues were collected from patients (31 male and 26 female patients; age range, 51-72 years) admitted to Weifang People's Hospital (Weifang, China) between May 2014 and February 2015. The study participants did not receive systemic or local anticancer treatments prior to surgical resection. All surgical tissues were immediately frozen and stored in liquid nitrogen for further use. The present study was approved by the Human Ethics Committee of Weifang People's Hospital and was performed in accordance with the Declaration of Helsinki. Written informed consent was obtained from all study participants.

Cell lines. A total of four NSCLC cell lines (SK-MES-1, H522, H460 and A549) and the human BEAS-2B nontumorigenic bronchial epithelial cell line were purchased from the American Type Culture Collection. SK-MES-1 cells were cultured in Minimum Essential Medium containing 10\% fetal bovine serum (FBS) and $1 \%$ penicillin-streptomycin (all Gibco; Thermo Fisher Scientific, Inc.). H522, H460, and A549 cells were cultured in RPMI 1640 (Gibco; Thermo Fisher Scientific, Inc.), though the other conditions were the same as those used for SK-MES-1 cells. BEAS-2B cells were cultured in Bronchial Epithelial Cell Growth Medium (Lonza Group Ltd.). All cells were maintained at $37^{\circ} \mathrm{C}$ in an incubator supplied with $5 \% \mathrm{CO}_{2}$.

Transfection. Specific small interfering RNAs (siRNAs) against CBR3-AS1 (si-CBR3-AS1\#1, \#2 and \#3) and a negative control siRNA (si-NC) were acquired from Guangzhou RiboBio Co., Ltd. The si-CBR3-AS1 sequences were as follows: si-CBR3-AS1\#1, 5'-ATGCAATTTCTTTAAAAA
GC-3'; si-CBR3-AS1\#2, 5'-CAGTTTATTTTTATTTAT TTTTT-3'; and si-CBR3-AS1\#3, 5'-AGCTCAAATTTTTTA TATATTTC-3'. The si-NC sequence was 5'-CACGATAAG ACAATGTATTT-3'. Furthermore, the miR-509-3p agomir (agomir-509-3p), negative control agomir (agomir-NC), miR-509-3p antagomir (antagomir-509-3p) and negative control antagomir (antagomir-NC) were synthesized by Shanghai GenePharma Co., Ltd. The corresponding sequences were as follows: Agomir-509-3p, 5'-GAUGGGUGUCUGCAU GGUUAGU-3'; agomir-NC, 5'-UUGUACUACACAAAAGUA CUG-3'; antagomir-509-3p, 5'-CUACCCACAGACGUACCA AUCA-3'; and antagomir-NC 5'-CAGUACUUUUGUGUA GUACAA-3'. The histone deacetylase 9 (HDAC9) overexpression vector pcDNA3.1-HDAC9 and empty pcDNA3.1 plasmid were purchased from Sangon Biotech Co., Ltd.

Cells were seeded into 6-well plates at a density of $6 \times 10^{5}$ cells per well, and then separately or co-transfected with the aforementioned siRNAs (100 pmol), agomirs (50 $\mathrm{nM})$, antagomirs $(100 \mathrm{nM})$ and plasmids $(4 \mu \mathrm{g})$ using Lipofectamine ${ }^{\circledR} 2000$ (Invitrogen; Thermo Fisher Scientific, Inc.). All transfection procedures were conducted at room temperature. After $48 \mathrm{~h}$ incubation at $37^{\circ} \mathrm{C}$, reverse transcription-quantitative (RT-q) PCR, flow cytometry and Transwell migration and invasion assays were carried out. Cell Counting kit-8 (CCK-8) and western blot assays were performed at 24 and $72 \mathrm{~h}$ post-transfection, respectively.

Nuclear-cytoplasmic fractionation, RNA isolation and $R T-q P C R$. The nuclear and cytoplasmic fractions of NSCLC cells were separated using the PARIS ${ }^{\mathrm{TM}}$ Kit (Invitrogen; Thermo Fisher Scientific, Inc). Next, the total nuclear and cytoplasmic RNA was extracted using TRIzol ${ }^{\circledR}$ reagent (Invitrogen; Thermo Fisher Scientific, Inc.), after which the RNA was subjected to RT-qPCR to determine the subcellular localization of CBR3-AS1. To quantify CBR3-AS1 and HDAC9 mRNA expression, complementary DNA was synthesized using the PrimeScript RT reagent Kit (Takara Biotechnology Co., Ltd.) and qPCR was conducted using SYBR Premix Ex Taq (Takara Biotechnology Co., Ltd.). The qPCR thermocycling conditions were as follows: $5 \mathrm{~min}$ at $95^{\circ} \mathrm{C}$, followed by 40 cycles of $95^{\circ} \mathrm{C}$ for $30 \mathrm{sec}$ and $65^{\circ} \mathrm{C}$ for $45 \mathrm{sec}$. GAPDH was used for the normalization of CBR3-AS1 and HDAC9 mRNA expression. To quantify miR-509-3p expression, total RNA was reversed transcribed using the miScript reverse transcription kit and qPCR was performed using the miScript SYBR Green PCR kit (both Qiagen $\mathrm{GmbH})$. The thermocycling conditions were as follows: $95^{\circ} \mathrm{C}$ for $2 \mathrm{~min}, 95^{\circ} \mathrm{C}$ for $10 \mathrm{sec}, 55^{\circ} \mathrm{C}$ for $30 \mathrm{sec}$ and $72^{\circ} \mathrm{C}$ for $30 \mathrm{sec}$, for 40 cycles. The expression of miR-509-3p was normalized to that of U6 small nuclear RNA. All expression levels were quantified using the $2^{-\Delta \Delta \mathrm{Cq}}$ method (22). Each group contained three replicates and RT-qPCR was repeated three times. The qPCR primer sequences were as follows: CBR3-AS1 forward, 5'-CAA TAGGGAAGCAGAGGGAGAA-3' and reverse, 5'-TTAGAG ATTCCTACAGACCCAGGTC-3'; HDAC9 forward, 5'-TCA GCTCAGTGGATGTGAAGTCA-3' and reverse, 5'-GCTGTT TCTGAAACTCTGCTATCAG-3'; GAPDH forward, 5'-CGG AGTCAACGGATTTGGTCGTAT-3' and reverse, 5'-AGCCTT CTCCATGGTGGTGAAGAC-3'; miR-509-3p forward, 5'-TCG GCAGGUACUGCAGACGUG-3' and reverse, 5'-CACTCA ACTGGTGTCGTGGA-3'; and U6 forward, 5'-GCTTCGGCA 
GCACATATACTAAAAT-3' and reverse, 5'-CGCTTCACG AATTTGCGTGTCAT-3'.

CCK- 8 assay. A total of $2 \times 10^{3} /$ well transfected cells resuspended in $100 \mu \mathrm{l}$ culture medium were inoculated into 96-well plates. The cells were incubated for $0,24,48$ or $72 \mathrm{~h}$, and $10 \mu \mathrm{l}$ of CCK-8 solution (Sigma-Aldrich, Merck KGaA) was added to each well, after which the plates were incubated at $37^{\circ} \mathrm{C}$ $\left(5 \% \mathrm{CO}_{2}\right)$ for an additional $2 \mathrm{~h}$. Absorbance was measured at $450 \mathrm{~nm}$ on a microplate reader (Multiscan MK3; Thermo Fisher Scientific, Inc.). Each group contained five replicates and the assay was repeated three times.

Flow cytometry. The Annexin V-Fluorescein Isothiocyanate (FITC) Apoptosis Detection Kit (BioLegend, Inc.) was used to determine apoptotic rate. Briefly, transfected cells were collected $48 \mathrm{~h}$ post-transfection and washed twice with ice-cooled phosphate-buffered solution (Gibco; Thermo Fisher Scientific, Inc.). The cells were centrifugated at $800 \mathrm{x}$ g for $5 \mathrm{~min}$ at room temperature, and resuspended in $100 \mu \mathrm{l}$ binding buffer. Then, $5 \mu 1$ Annexin V-FITC and $10 \mu l$ propidium iodide were added for double staining. Following a 15-min incubation in the dark at room temperature, the apoptotic rate was determined by flow cytometry (FACScan; BD Biosciences). CellQuest software (version 2.9; BD Biosciences) was used for data analysis. Each group contained three replicates and the assay was repeated three times.

Transwell migration and invasion assays. Transfected cells were harvested and resuspended in serum-free culture medium. Next, $200 \mu \mathrm{l}$ cell suspension $\left(5 \times 10^{4}\right.$ cells) was added into the upper compartments of Transwell inserts (pore size, $8 \mu \mathrm{m}$; BD Biosciences) and $600 \mu \mathrm{l}$ complete culture medium was added to the bottom chambers to induce migration. After $24 \mathrm{~h}$ incubation at $37^{\circ} \mathrm{C}$, non-migratory cells were removed from the upper chamber using a cotton swab, and cells that had migrated to the lower chamber were fixed with methanol at room temperature for $30 \mathrm{~min}$ and stained with $0.1 \%$ crystal violet at room temperature for $30 \mathrm{~min}$. Following extensive rinsing, imaging of the stained cells was performed using an inverted microscope (Olympus Corporation; x200 magnification), and five randomly selected fields were counted. The same procedure was followed for the Transwell invasion assay, but the membranes were precoated with Matrigel (BD Biosciences) at $37^{\circ} \mathrm{C}$ for $2 \mathrm{~h}$. The number of migratory and invasive cells was counted, and the average values were used to determine the migratory and invasive capacities, respectively. The assay was repeated three times.

Tumor xenograft model. Plasmids carrying short hairpin RNA (shRNA) against CBR3-AS1 (pLKO.1-sh-CBR3-AS1) or the negative control shRNA (pLKO.1-sh-NC) were manufactured by Shanghai GenePharma Co.,Ltd.H460 cells were transfected with the aforementioned lentivirus produced by Shanghai GenePharma Co., Ltd, and puromycin was used to select a stable CBR3-AS1-knockdown cell line. Animal experimental procedures were approved by the Institutional Animal Care and Use Committee of Weifang People's Hospital. For the xenograft study, H460 cells stably transfected with sh-CBR3-AS1 (sh-CBR3-AS1 group) or sh-NC (sh-NC group) were collected and subcutaneously injected into 4-6-week-old male BALB/c nude mice (20 g each; Beijing Vital River Laboratory Animal Technology Co., Ltd). A total of six mice were used in the assay (three mice per group). The animals were maintained under specific pathogen-free conditions at $25^{\circ} \mathrm{C}$ and $50 \%$ humidity, with a 10:14 light/dark cycle and ad libitum access to food and water. The size of the subcutaneous tumors was monitored and recorded weekly for 4 weeks. Tumor volume was calculated as follows: Volume $=0.5 \mathrm{x}$ length $\mathrm{x}$ width $^{2}$. All mice were euthanized by cervical dislocation after the last measurement, and the subcutaneous tumors were resected and weighed. Tumor xenografts were collected and used for RT-qPCR and western blot analysis.

Bioinformatics analysis. The potential interaction between CBR3-AS1 and miRNA(s) was predicted using the StarBase online tool (http://starbase.sysu.edu.cn/). For miRNA target prediction, two online databases, miRDB (http://mirdb.org/) and TargetScan (http://www.targetscan.org/vert_72/), were used to search for putative targets of miR-509-3p. IncLocator (http://www.csbio.sjtu.edu.cn/bioinf/lncLocator/) was utilized to predict the localization of CBR3-AS1.

Luciferase reporter assay. Partial sequences of CBR3-AS1 carrying wild-type (WT) miR-509-3p binding sequences were amplified and inserted into the pmirGLO Dual-Luciferase miRNA Target Expression Vector (Promega Corporation) and termed WT-CBR3-AS1. The mutant (MUT) sequences of CBR3-AS1 were also cloned into the same vector to generate the MUT-CBR3-AS1 luciferase reporter vector. WT-HDAC9 and MUT-HDAC9 luciferase reporter vectors were designed and produced using the same procedure. NSCLC cells were seeded into 24-well plates and $24 \mathrm{~h}$ later, were co-transfected with WT or MUT luciferase reporter vectors along with agomir-509-3p or agomir-NC using Lipofectamine ${ }^{\circledR} 2000$. After $48 \mathrm{~h}$, luciferase activity was measured using the Dual-Glo ${ }^{\circledR}$ Luciferase Assay System (Promega Corporation). The assay was repeated three times and contained three replicates. Renilla luciferase activity was used for the normalization of firefly luciferase activity.

RNA immunoprecipitation (RIP). The Magna RIP ${ }^{\mathrm{TM}}$ RNA-Binding Protein Immunoprecipitation kit (EMD Millipore) was used per the manufacturer's protocol to determine potential interactions between CBR3-AS1 with miR-509-3p in NSCLC. NSCLC cells were harvested and lysed using the supplied RIP lysis buffer. Then, whole-cell extracts were incubated with RIP buffer and human anti-Argonaute (anti-Ago2) or anti-IgG antibodies (both cat. no. 03-110; EMD Millipore). After overnight incubation, the magnetic beads were collected, rinsed with washing buffer and treated with proteinase $\mathrm{K}$ to digest the proteins. The immunoprecipitated RNA was analyzed by RT-qPCR. The RIP assay was repeated three times and contained three replicates.

Protein preparation and western blotting. Cells were lysed in RIPA buffer (Beyotime Institute of Biotechnology) supplemented with a protease inhibitor cocktail (Roche Diagnostics), and the extracted protein was quantified using a bicinchoninic acid protein assay kit (Nanjing KeyGen Biotech Co., Ltd.). 
A

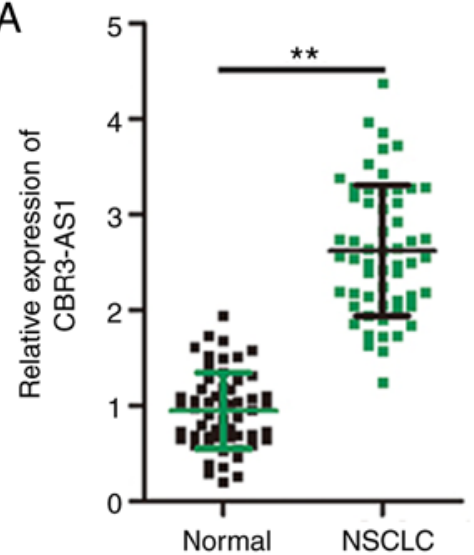

B

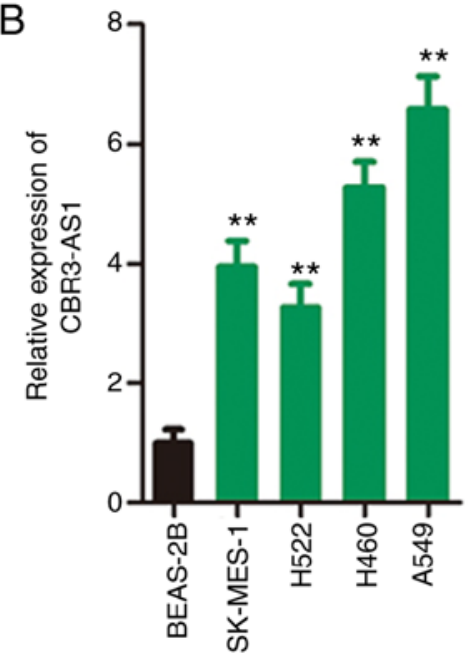

C

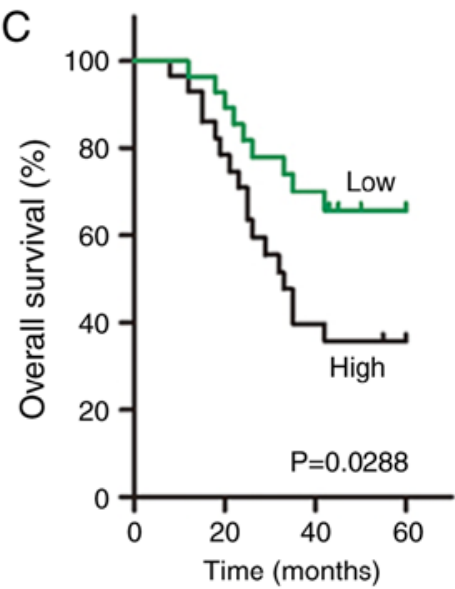

Figure 1. CBR3-AS1 expression is upregulated in NSCLC and is closely associated with poor clinical outcome. (A) Relative CBR3-AS1 expression was detected using RT-qPCR in 57 pairs of NSCLC and adjacent healthy tissues. (B) RT-qPCR was performed to characterize the expression pattern of CBR3-AS1 in four NSCLC cell lines. The human nontumorigenic bronchial epithelium cell line BEAS-2B served as a control. (C) Overall survival was assessed using Kaplan-Meier survival plots according to CBR3-AS1 expression in NSCLC patients $(\mathrm{P}=0.0288)$. ${ }^{* *} \mathrm{P}<0.01$. CBR3-AS1, CBR3 antisense RNA 1; NSCLC, non-small cell lung cancer; RT-qPCR, reverse transcription-quantitative PCR.

Equal amounts of protein (30 $\mu \mathrm{g}$ per well) were separated by electrophoresis on $10 \%$ SDS-PAGE gels, and transferred onto polyvinylidene difluoride membranes (EMD Millipore). The membranes were blocked for $2 \mathrm{~h}$ at room temperature with $5 \%$ nonfat dry milk in Tris-buffered saline $(0.1 \%$ Tween-20). After blocking, the membranes were incubated with primary antibodies [anti-HDAC9 (cat. no. ab109446; Abcam) and anti-GAPDH (cat. no. ab128915; Abcam), both $1: 1,000]$ at $4^{\circ} \mathrm{C}$ overnight, and further incubated with a horseradish peroxidase-conjugated secondary antibody (1:5,000; cat. no. ab205718; Abcam). The blots were developed using the Immobilon Western Chemilum HRP substrate (EMD Millipore) and the assay was repeated three times. Quantity One software version 4.62 (Bio Rad Laboratories, Inc.) was used for densitometric analysis.

Statistical analysis. The experimental results were analyzed using the SPSS statistics software package (version 21.0; IBM Corp) and expressed as the mean \pm standard deviation. The $\chi^{2}$ test was used to evaluate the association between CBR3-AS1 expression and the clinicopathological characteristics of patients with NSCLC. Differences in CBR3-AS1 expression between tissue samples were assessed using paired Student's $t$-tests. One-way analysis of variance followed by Tukey's post-hoc test was performed to compare differences among multiple groups. Correlations between the expression levels of CBR3-AS1 and miR-509-3p in NSCLC tissues were analyzed using Spearman's correlation analysis. Patient overall survival was analyzed using Kaplan-Meier survival analysis, and the results were compared using the log-rank test. $\mathrm{P}<0.05$ was considered to indicate a statistically significant difference.

\section{Results}

CBR3-AS1 is upregulated and closely associated with poor prognosis in NSCLC. In total, 57 pairs of NSCLC tissues and adjacent healthy tissues were collected during the present study, and RT-qPCR was performed to determine CBR3-AS1 expression. CBR3-AS1 expression was prominently upregulated in NSCLC tissues compared with that in adjacent healthy tissues (Fig. 1A). CBR3-AS1 expression was also consistently higher in all four tested NSCLC cell lines (SK-MES-1, H522, $\mathrm{H} 460$ and A549) than in the human BEAS-2B nontumorigenic bronchial epithelium cell line (Fig. 1B).

To determine the clinical relevance of CBR3-AS1 in NSCLC, the patients were divided into high ( $\geq$ median) or low (< median) CBR3-AS1 expression groups based on the median CBR3-AS1 level of their NSCLC tissue samples. $\chi^{2}$ analysis revealed that among the 57 patients, increased CBR3-AS1 expression was closely correlated with larger tumor size $(\mathrm{P}=0.033)$, advanced TNM stage $(\mathrm{P}=0.014)$, and increased incidence of lymph node metastasis $(\mathrm{P}=0.024$; Table I). Furthermore, Kaplan-Meier survival analysis revealed a significantly shorter overall survival time in NSCLC patients exhibiting high CBR3-AS1 expression than in those with low CBR3-AS1 expression (Fig. 1C; $\mathrm{P}=0.0288$ ). Collectively, these findings indicate aberrant CBR3-AS1 expression in NSCLC, which is strongly correlated with tumorigenesis and progression.

CBR3-AS1-knockdown inhibits the aggressive phenotypes of NSCLC cells. Given the expression profile of CBR3-AS1 in NSCLC cell lines and tissues, it was next determined whether CBR3-AS1 was required for the malignant progression of NSCLC. To this end, three siRNAs against CBR3-AS1 were transfected into $\mathrm{H} 460$ and A549 cells, and knockdown efficiency was assessed by RT-qPCR. si-CBR3-AS1\#1 was shown to be the most effective at silencing CBR3-AS1 expression in both $\mathrm{H} 460$ and A549 cells (Fig. 2A) and was therefore used in the following functional assays, and is henceforth referred to as si-CBR3-AS1. CCK-8 assays indicated that CBR3-AS1-knockdown markedly inhibited proliferative ability (Fig. 2B), and flow cytometry revealed that CBR3-AS1-knockdown promoted apoptosis (Fig. 2C) in 
Table I. Correlations between CBR3-AS1 and the clinicopathological characteristics of 57 patients with NSCLC.

CBR3-AS1 expression

Clinicopathological characteristic

Sex

Male

Female

Age, years

$<60$

$\geq 60$

Tumor size, $\mathrm{cm}$

$<3$

$\geq 3$

Differentiation

High and Moderate

Poor

TNM stage

I+II

III+IV

Lymph node metastasis

Negative

Positive

$\overline{\text { High Low }}$

P-value

17

12

14

14

$14 \quad 12$

$15-16$

12

17

899

21

19

13

16

22

6

$0.024^{\mathrm{a}}$

15

14
23

5

${ }^{\mathrm{a}} \mathrm{P}<0.05$ by $\chi^{2}$ test.

A

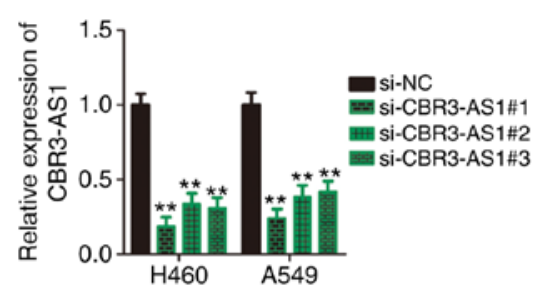

C

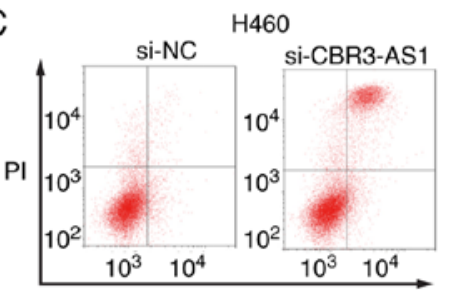

Annexin V
B
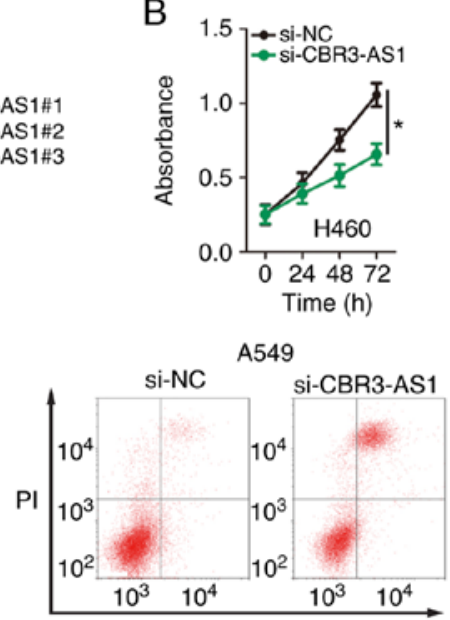

$\mathrm{E}$

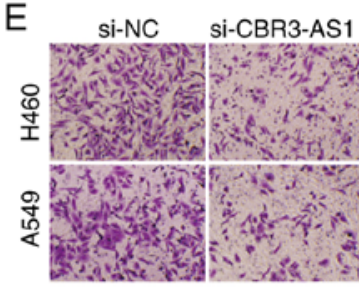

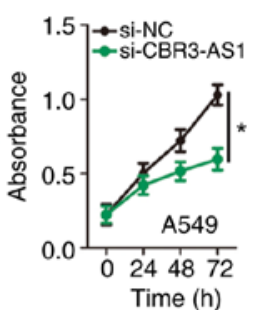

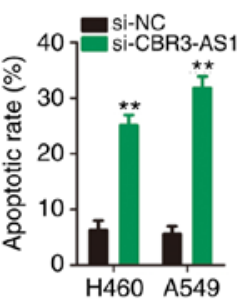

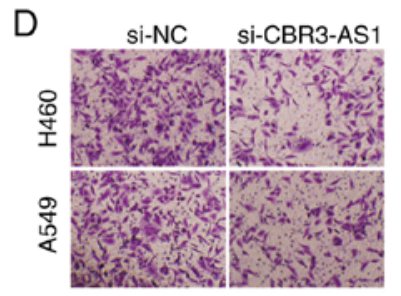
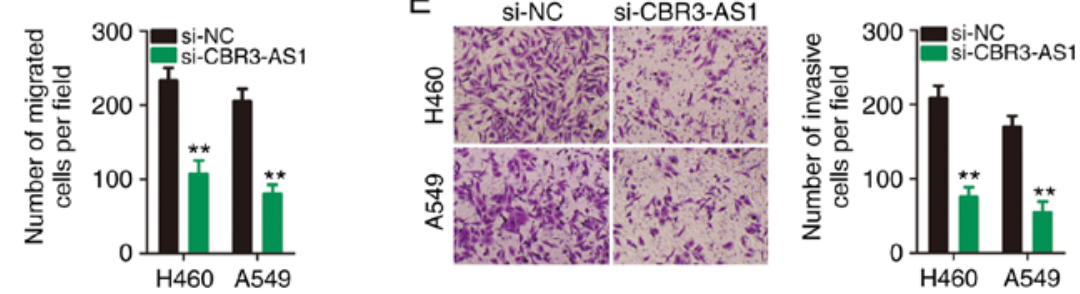

Figure 2. CBR3-AS1-knockdown inhibits cellular proliferation, migration and invasiveness, and promotes apoptosis in NSCLC. (A) CBR3-AS1 expression in H460 and A549 cells transfected with three CBR3-AS1 siRNAs (si-CBR3-AS1\#1, \#2 and \#3) and si-NC was measured using RT-qPCR. (B) Proliferation of CBR3-AS1-depleted H460 and A549 cells was determined using the Cell Counting Kit 8 assay. (C) Flow cytometry was performed to determine the apoptotic rates of $\mathrm{H} 460$ and A549 cells after CBR3-AS1 inhibition. The (D) migratory and (E) invasive abilities of si-CBR3-AS1- or si-NC-transfected H460 and A549 cells were evaluated by Transwell migration and invasion assays. Magnification, $\mathrm{x} 200$. $^{*} \mathrm{P}<0.05$ and ${ }^{* *} \mathrm{P}<0.01$. CBR3 antisense RNA 1 ; NSCLC, non-small cell lung cancer; RT-qPCR, reverse transcription-quantitative PCR; si(RNA), small interfering; NC, negative control. 

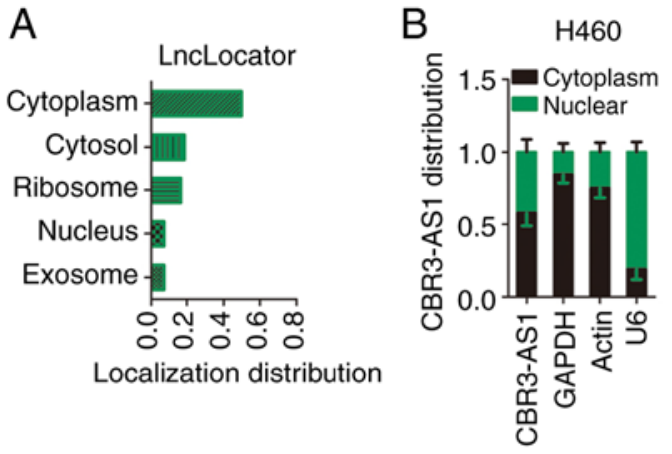
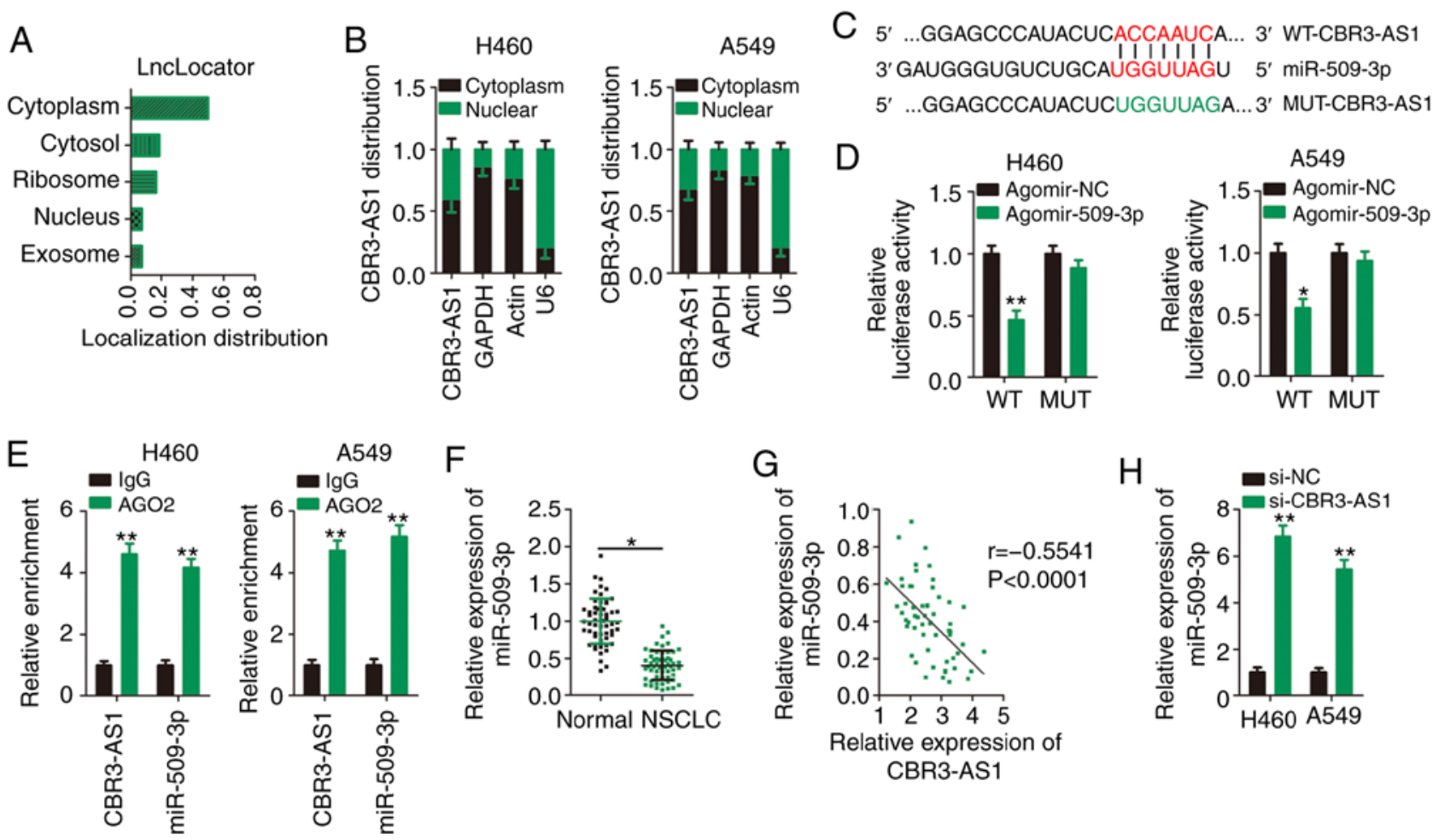

Figure 3. CBR3-AS1 acts as a competing endogenous RNA by interacting with miR-509-3p in NSCLC cells. (A) 1ncLocator was used to predict the localization of CBR3-AS1. (B) Subcellular distribution of CBR3-AS1 was examined in H460 and A549 cells by nuclear-cytoplasmic fractionation and RT-qPCR. (C) Speculated complementary binding sequences of miR-509-3p in CBR3-AS1. (D) Luciferase reporter vectors carrying wild-type or mutant miR-509-3p-binding sites were co-transfected into H460 and A549 cells with agomir-509-3p or agomir-NC. (E) RNA immunoprecipitation assays were performed in H460 and A549 cells, and the enrichment of CBR3-AS1 and miR-509-3p in the coprecipitated RNA was quantified by RT-qPCR. (F) Expression patterns of miR-509-3p in 57 pairs of NSCLC and adjacent healthy tissues were determined by RT-qPCR. (G) Spearman's correlation analysis to determine the association between CBR3-AS1 and miR-509-3p expressions in 57 paired tissue samples $(\mathrm{r}=-0.5541, \mathrm{P}<0.0001)$. (H) Influence of CBR3-AS1-knockdown on miR-509-3p expression in H460 and A549 cells was evaluated by RT-qPCR. ${ }^{*} \mathrm{P}<0.05$ and ${ }^{* *} \mathrm{P}<0.01$. CBR3 antisense RNA 1 ; NSCLC, non-small cell lung cancer; RT-qPCR, reverse transcription-quantitative PCR; miR, microRNA; NC, negative control.

H460 and A549 cells. Furthermore, the migration (Fig. 2D) and invasion (Fig. 2E) capacities of CBR3-AS1-deficient H460 and A549 cells were hindered compared with cells transfected with si-NC. Taken together, these data indicate that the downregulation of CBR3-AS1 suppressed proliferation, migration and invasiveness and promoted apoptosis in NSCLC cells.

CBR3-AS1 acts as a ceRNA and sponges miR-509-3p in NSCLC cells. To determine the molecular events implicated in the CBR3-AS1-assocaited control of aggressive NSCLC cell phenotypes, IncLocator (http://www.csbio.sjtu. edu.cn/bioinf/lncLocator/), an IncRNA subcellular localization predictor, was used to predict the cellular localization of CBR3-AS1. CBR3-AS1 was predicted to be predominantly located in the cytoplasm (Fig. 3A), which was similar to the results of nuclear-cytoplasmic fractionation (Fig. 3B). These results suggest that $\mathrm{CBR} 3-\mathrm{AS} 1$ regulates the expression of target proteins at the post-transcriptional level. An increasing number of studies has illustrated that cytoplasmic lncRNAs function as ceRNAs or molecular sponges for specific miRNAs, thereby liberating miRNAs from their target RNA transcripts (23).

Bioinformatics analysis was performed to identify miRNA(s) that could directly interact with CBR3-AS1. miR-509-3p was predicted to contain putative complementary binding sequences for CBR3-AS1 (Fig. 3C). Because miR-509-3p plays a crucial role in tumorigenesis and cancer progression (24-27), it was selected for further analysis. As is evident from the luciferase reporter assay, miR-509-3p overexpression successfully suppressed the luciferase activity of the reporter vector (harboring WT miR-509-3p binding sites) in H460 and A549 cells (Fig. 3D), and this suppression was abrogated when the miR-509-3p binding sequences were mutated (MUT-CBR3-AS1). Next, a RIP assay was used to determine whether CBR3-AS1 and miR-509-3p are present in the RNA-induced silencing complex. The results indicated that compared with the IgG control group, CBR3-AS1 and miR-509-3p were notably enriched in the Ago2-containing magnetic beads in H460 and A549 cells (Fig. 3E).

To further elucidate the correlation between CBR3-AS1 and miR-509-3p expression, RT-qPCR was performed to determine the expression levels of miR-509-3p in 57 paired NSCLC and adjacent healthy tissues. miR-509-3p expression was downregulated in NSCLC tissues compared with adjacent healthy tissues (Fig. 3F), which was inversely correlated with that of CBR3-AS1 in NSCLC tissues (Fig. 3G; r=-0.5541, P<0.0001). Furthermore, miR-509-3p expression was increased following CBR3-AS1-knockdown in H460 and A549 cells (Fig. 3H). Collectively, CBR3-AS1 was shown to act as a molecular sponge that inhibits miR-509-3p expression in NSCLC cells. 

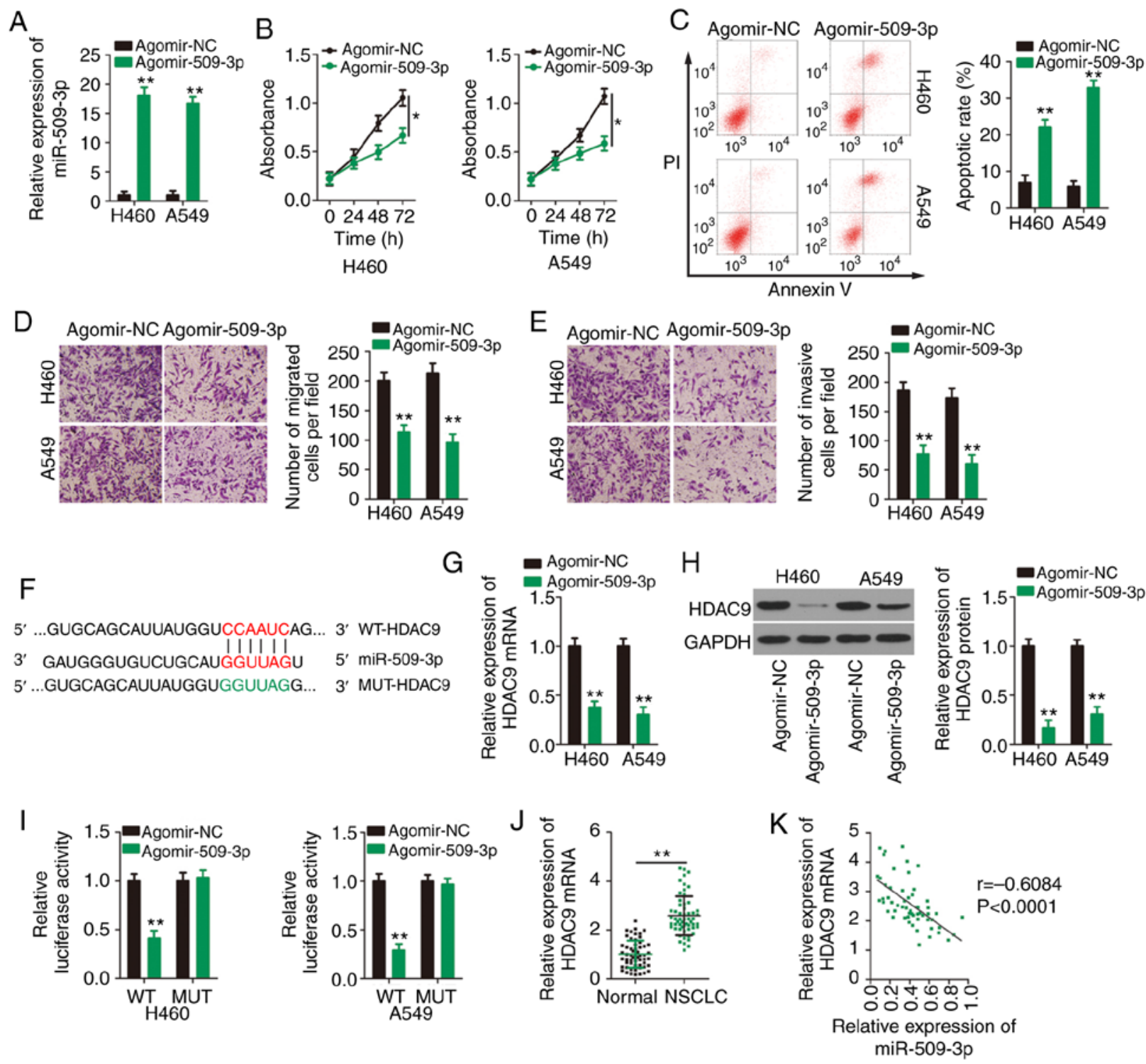

Figure 4. miR-509-3p directly targets HDAC9 in NSCLC cells. (A) Agomir-509 or agomir-NC was transfected into H460 and A549 cells and transfection efficiency was validated using RT-qPCR. (B) Proliferation and (C) apoptosis of $\mathrm{H} 460$ and A549 cells transfected with agomir-509 or agomir-NC were detected by Cell Counting Kit 8 assays and flow cytometry, respectively. Transwell (D) migration and (E) invasion assays were performed to assess the effects of miR-509-3p overexpression on the migratory and invasive abilities of H460 and A549 cells. Magnification, x200. (F) WT and MUT miR-509-3p target sites in the HDAC9 transcript. (G) RT-qPCR and (H) western blotting were performed to detect HDAC9 mRNA and protein expression, respectively, in H460 and A549 cells after upregulation of miR-509-3p expression. (I) Luciferase reporter assays were performed to determine the luciferase activities of H460 and A549 cells after co-transfection with agomir-509 or agomir-NC and WT-HDAC9 or MUT-HDAC9. (J) RT-qPCR for the expression of HDAC9 mRNA in 57 pairs of NSCLC and adjacent healthy tissues. (K) Spearman's correlation analysis was performed to analyze the correlation between miR-509-3p and HDAC9 mRNA expression in 57 NSCLC tissues $(\mathrm{r}=-0.6084, \mathrm{P}<0.0001)$. ${ }^{*} \mathrm{P}<0.05$ and ${ }^{* *} \mathrm{P}<0.01$. miR, microRNA; HDAC9, histone deacetylase 9; NSCLC, non-small cell lung cancer; NC, negative control; RT-qPCR, reverse transcription-quantitative PCR; WT, wild-type; MUT, mutant.

HDAC9 is a direct target gene of miR-509-3p in NSCLC cells. To assess the role of CBR3-AS1 in NSCLC, agomir-509-3p or agomir-NC were transfected into $\mathrm{H} 460$ and A549 cells. RT-qPCR analysis confirmed that miR-509-3p expression was markedly increased in both cell lines following agomir-509-3p transfection (Fig. 4A). CCK-8 assay and flow cytometric assays revealed that exogenous miR-509-3p expression significantly restrained proliferation (Fig. 4B) and promoted apoptosis (Fig. 4C) in $\mathrm{H} 460$ and A549 cells. Furthermore, Transwell migration and invasion assays confirmed that ectopic miR-509-3p expression reduced the migratory (Fig. 4D) and invasive (Fig. 4E) abilities of H460 and A549 cells.
To investigate the mechanisms underlying the tumor-suppressing roles of miR-509-3p in NSCLC cells, online bioinformatics tools were used to predict the potential targets of miR-509-3p. HDAC9 was predicted to contain a putative binding site for miR-509-3p (Fig. 4F). RT-qPCR and western blotting were then performed to analyze the impacts of miR-509-3p upregulation on HDAC9 mRNA and protein expression, respectively. Transfection with agomir-509-3p resulted in a significant decrease in HDAC9 expression at the both mRNA (Fig. 4G) and protein (Fig. 4H) levels. Next, a luciferase reporter assay was performed to address the direct binding between miR-509-3p and HDAC9 in NSCLC cells. Overexpression of miR-509-3p reduced the luciferase 
A
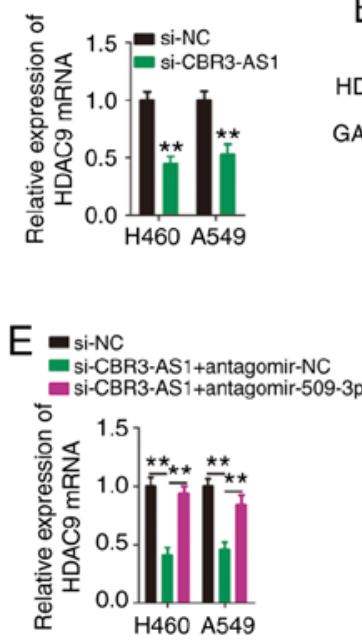

B
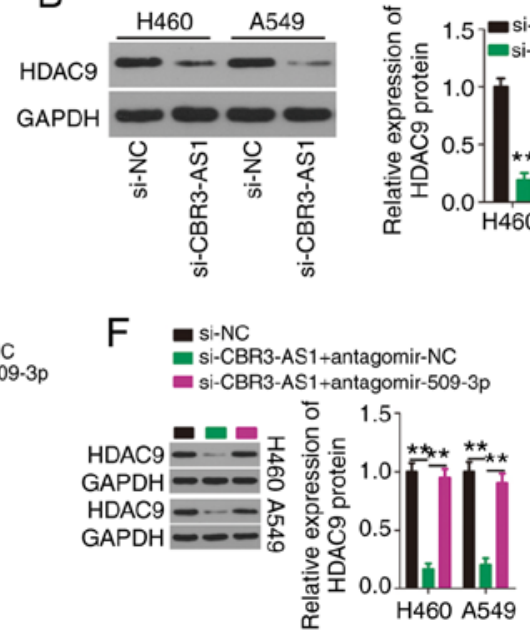

$\mathrm{C}$

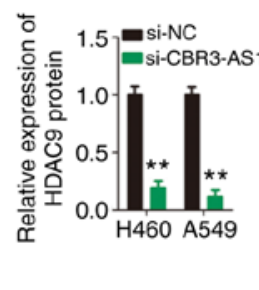

(1)

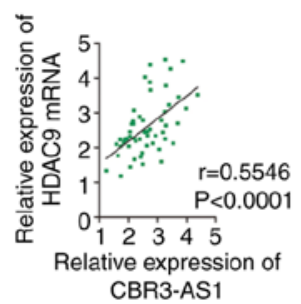

$\begin{aligned} G & \rightarrow \text { si-NC } \\ & \rightarrow \text { si-CBR3-AS1+antagomir-NC } \\ & \rightarrow \text { si-CBR3-AS1+antagomir-509-3p }\end{aligned}$

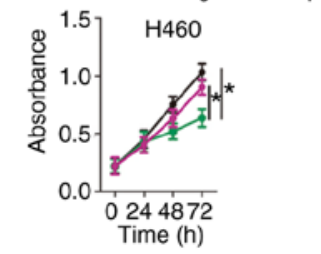

D

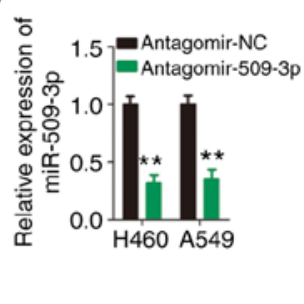

$\rightarrow$ si-NC

- si-CBR3-AS1+antagomir-NC

- si-CBR3-AS1+antagomir-509-3p

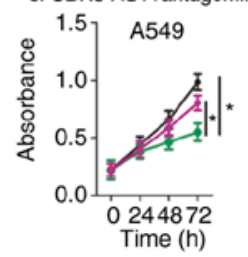

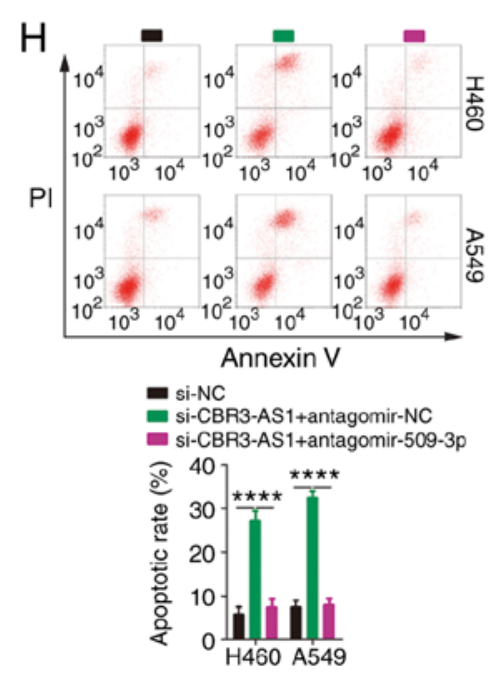
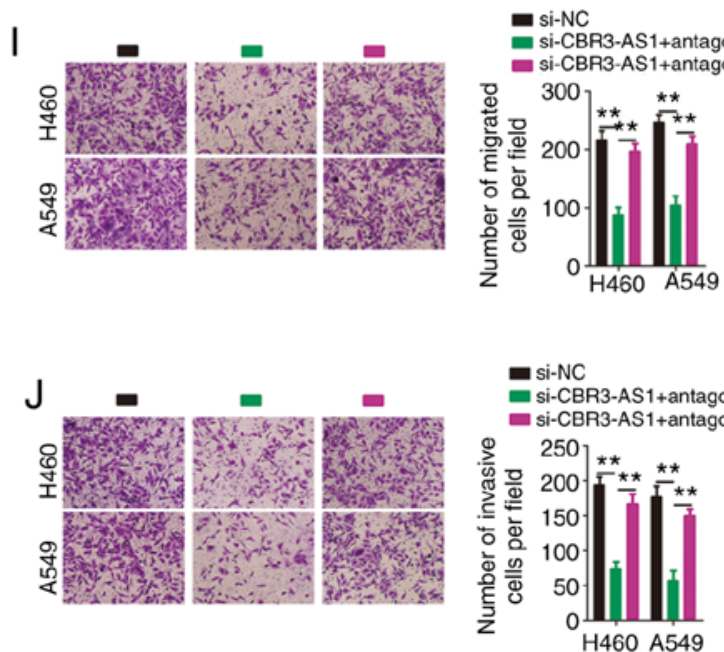

Figure 5. Suppression of miR-509-3p expression counteracts the effects of CBR3-AS1 knockdown on NSCLC cells. H460 and A549 cells were transfected with si-CBR3-AS1 or si-NC, and HDAC9 mRNA and protein expression were measured by (A) RT-qPCR and (B) western blotting, respectively. (C) Correlation between CBR3-AS1 and HDAC9 mRNA expression in 57 paired NSCLC tissues was examined using Spearman's correlation analysis $(\mathrm{r}=0.5546, \mathrm{P}<0.0001)$. (D) Silencing efficiency of antagomir-509-3p in H460 and A549 cells was evaluated by RT-qPCR. Antagomir-NC acted as the control for antagomir-509-3p. CBR3-AS1-deficient H460 and A549 cells were transfected with antagomir-509-3p or antagomir-NC. (E) RT-qPCR and (F) western blotting were used to assess changes in HDAC4 mRNA and protein expression, respectively. (G) Proliferation and (H) apoptosis were detected by Cell Counting Kit 8 and flow cytometric assays, respectively. Transwell (I) migration and $(\mathrm{J})$ invasion assays were performed to determine the migratory and invasive abilities of transfected H460 and A549 cells. Magnification, $\mathrm{x} 200 .{ }^{*} \mathrm{P}<0.05,{ }^{* *} \mathrm{P}<0.01$ and ${ }^{* * * *} \mathrm{P}<0.0001$. miR, microRNA; CBR3 antisense RNA 1; NSCLC, non-small cell lung cancer; si(RNA), small interfering; NC, negative control; HDAC9, histone deacetylase 9; RT-qPCR, reverse transcription-quantitative PCR.

activity of WT-HDAC9 in H460 and A549 cells, but not that of its mutated counterpart (Fig. 4I). Further analysis confirmed that HDAC9 was highly expressed in NSCLC tissues compared with adjacent healthy tissues (Fig. 4J), and a negative expression correlation between HDAC9 mRNA and miR-509-3p was identified in NSCLC tissues (Fig. 4K; $\mathrm{r}=-0.6084, \mathrm{P}<0.0001)$. The aforementioned results collectively confirm that HDAC9 is a direct target of miR-509-3p in NSCLC cells.

CBR3-ASI drives the tumorigenicity of NSCLC cells through the miR-509-3p/HDAC9 axis. In the present study, CBR3-AS1 was found to function as a ceRNA for miR-509-3p, and HDAC9 as a direct target of miR-509-3p in NSCLC cells; accordingly, whether CBR3-AS1 positively modulates HDAC9 expression in NSCLC via miR-509-3p sponging was subsequently assessed.
Silencing of CBR3-AS1 decreased HDAC9 mRNA (Fig. 5A) and protein (Fig. 5B) expression in both $\mathrm{H} 460$ and A549 cells. Consistently, CBR3-AS1 expression was positively correlated with HDAC9 mRNA expression in 57 NSCLC tissue samples (Fig. 5C; $r=0.5546, \mathrm{P}<0.0001$ ). Rescue experiments were then performed to determine whether miR-509-3p sponging was required for the CBR3-AS1-mediated regulation of HDAC9. Antagomir-509-3p was used to silence miR-509-3p expression in $\mathrm{H} 460$ and A549 cells, and silencing efficiency was verified by RT-qPCR (Fig. 5D). Antagomir-509-3p or antagomir-NC (in combination with si-CBR3-AS1) were co-transfected into H460 and A549 cells, and changes in HDAC9 expression were evaluated. In H460 and A549 cells, CBR3-AS1-knockdown decreased HDAC9 mRNA (Fig. 5E) and protein (Fig. 5F) expression, which was mostly restored after co-transfection with antagomir-509-3p. 
A

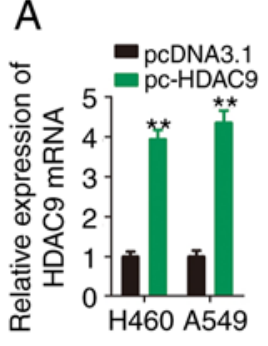

B

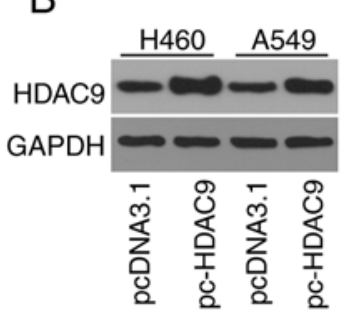

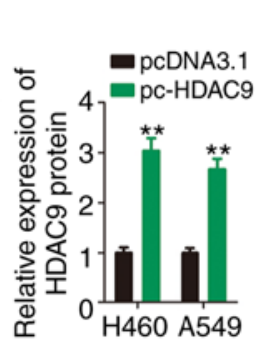

C $\rightarrow$ Si-NC $\rightarrow$ Si-CBR3-AS1+PCDNA3.1 $\rightarrow$ si-CBR3-AS1+pC-HDAC9

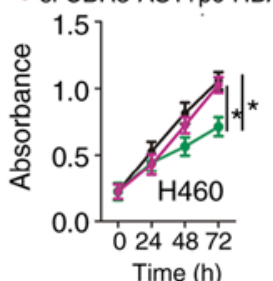

E

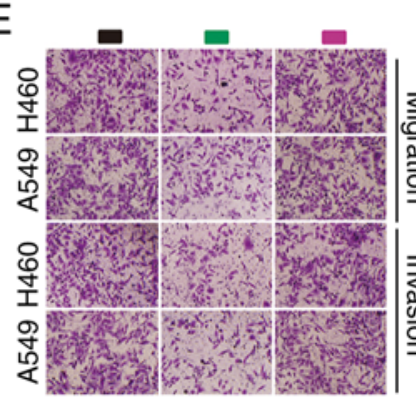

$\rightarrow$ si-NC

- si-CBR3-AS1+pcDNA3.1

$\rightarrow$ si-CBR3-AS1+pc-HDAC9

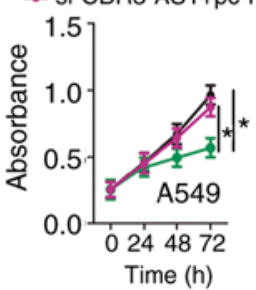

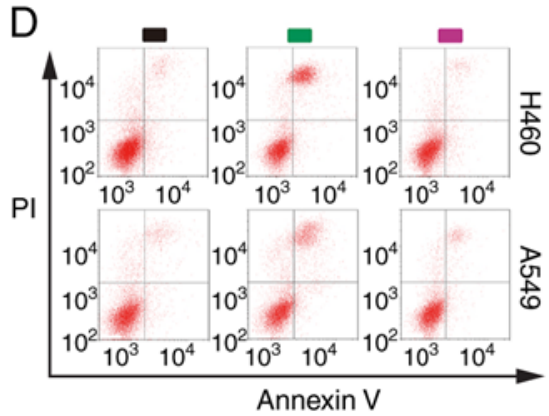
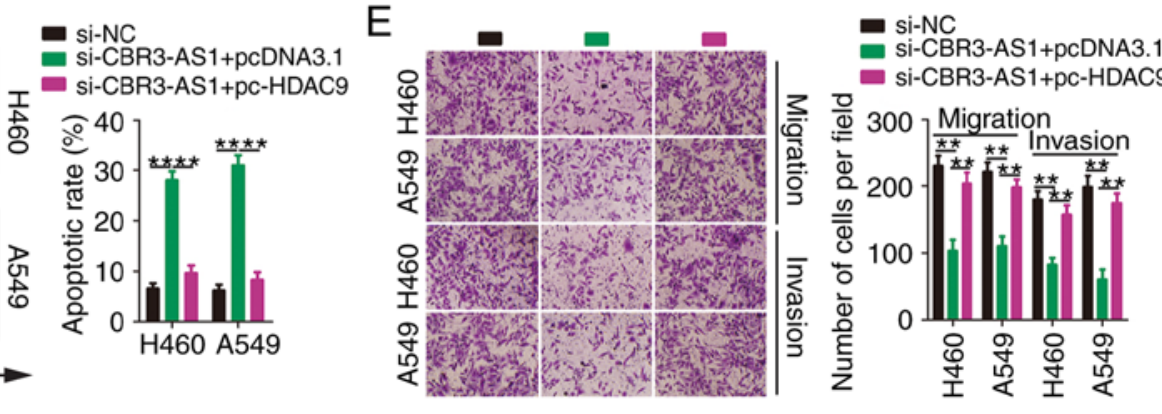

Figure 6. Effects of CBR3-AS1-knockdown on NSCLC cells are neutralized by HDAC9 reintroduction. (A) HDAC9 mRNA and (B) protein expression were assessed by reverse transcription-quantitative PCR and western blotting, respectively, in H460 and A549 cells after pcDNA3.1 or pc-HDAC9 transfection. si-CBR3-AS1 in parallel with pcDNA3.1 or pc-HDAC9 was co-transfected into H460 and A549 cells. (C) Proliferation, (D) apoptosis, (E) migration and invasiveness were evaluated by Cell Counting Kit 8 assay, flow cytometry, and transwell migration and invasion assays, respectively. Magnification, x200. ${ }^{*} \mathrm{P}<0.05,{ }^{* *} \mathrm{P}<0.01$ and ${ }^{* * * *} \mathrm{P}<0.0001$. CBR3 antisense RNA 1; NSCLC, non-small cell lung cancer; HDAC9, histone deacetylase 9; si, small interfering.
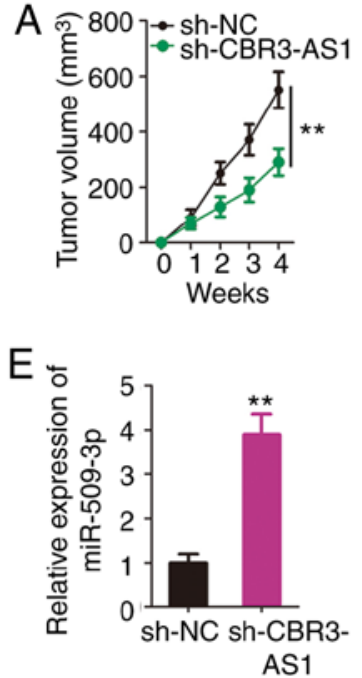

B
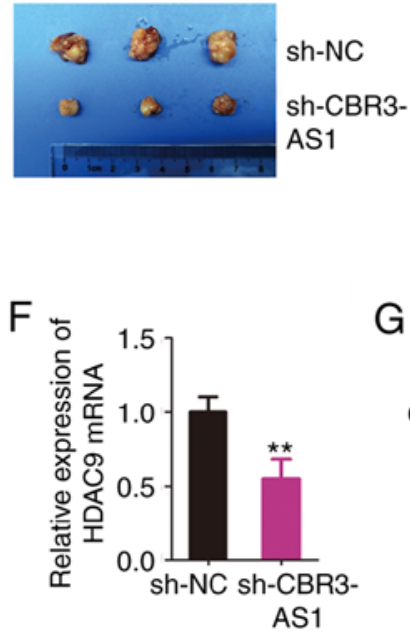
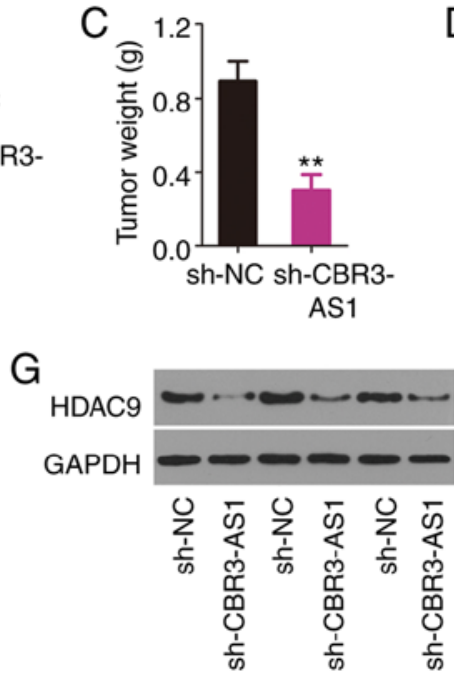
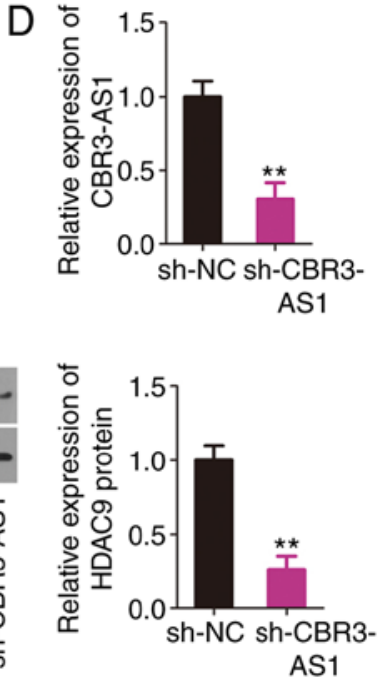

Figure 7. CBR3-AS1 silencing restricts NSCLC tumor growth in vivo. (A) Tumor growth curves were plotted by measuring tumor volume weekly for 4 weeks after the injection of $\mathrm{H} 460$ cells stably transfected with sh-CBR3-AS1 or sh-NC. (B) Representative images of subcutaneous tumors in nude mice 4 weeks after injection of H460 cells stably transfected with sh-CBR3-AS1 or sh-NC. (C) Weights of subcutaneous tumors isolated from the sh-CBR3-AS1 and sh-NC groups were detected 4 weeks after cell inoculation. (D) CBR3-AS1 and (E) miR-509-3p expression in subcutaneous tumors was measured using RT-qPCR. (F) RT-qPCR and (G) western blotting were performed to analyze HDAC9 mRNA and protein levels in subcutaneous tumors, respectively. ${ }^{* *}$ P $<0.01$. CBR3 antisense RNA 1; NSCLC, non-small cell lung cancer; sh, short hairpin; NC, negative control; RT-qPCR reverse transcription-quantitative PCR; HDAC9, histone deacetylase 9.

CCK-8 and flow cytometric assays showed that downregulating CBR3-AS1 expression restricted $\mathrm{H} 460$ and A549 cell proliferation (Fig. 5G) and promoted apoptosis (Fig. 5H), which were abolished by co-transfecting cells with antagomir-509-3p. Similarly, decreased CBR3-AS1 expression impaired the migratory (Fig. 5I) and invasive (Fig. 5J) abilities of $\mathrm{H} 460$ and A549 cells, and these effects were abrogated by synergistically knocking down miR-509-3p expression.
Rescue experiments were also conducted to determine whether upregulating HDAC9 reversed the malignant phenotypes of NSCLC cells in conjunction with CBR3-AS1-knockdown. HDAC9 overexpression increased both HDAC9 mRNA (Fig. 6A) and protein (Fig. 6B) levels in $\mathrm{H} 460$ and A549 cells. Next, pc-HDAC9 or pcDNA3.1 were introduced into $\mathrm{H} 460$ and A549 cells in the presence of si-CBR3-AS1.Functionally, theimpactsofCBR3-AS1 inhibition 
on the proliferation (Fig. 6C), apoptosis (Fig. 6D), migration (Fig.6E) and invasiveness (Fig. 6E) of H460 and A549 cells were counteracted by HDAC 9 overexpression. Together, these data indicate that the miR-509-3p/HDAC9 axis mediates the cancer-promoting effects of CBR3-AS1 in NSCLC cells.

CBR3-AS1 interference impedes NSCLC tumor growth in vivo. A tumor xenograft model was established to determine tumor growth after a subcutaneous injection of H460 cells stably transfected with sh-CBR3-AS1 or sh-NC. Tumor growth was inhibited in the sh-CBR3-AS1 group compared with that in the sh-NC group (Fig. 7A). After 4 weeks, all mice were euthanized and the subcutaneous tumors were resected and imaged (Fig. 7B). The average weight of the subcutaneous tumors was found to be decreased in the sh-CBR3-AS1 group compared with the sh-NC group (Fig. 7C). Furthermore, RT-qPCR was performed to indicate the changes in CBR3-AS1 and miR-509-3p expression in the sh-CBR3-AS1 and sh-NC group tumors. The results indicated that CBR3-AS1 expression was downregulated (Fig. 7D), whereas miR-509-3p expression was upregulated (Fig. 7E) in the sh-CBR3-AS1 group. Furthermore, the mRNA (Fig. 7F) and protein (Fig. 7G) levels of HDAC9 were evidently decreased in the sh-CBR3-AS1 group compared with those in the sh-NC group. Thus, these results indicate that CBR3-AS1-knockdown suppressed NSCLC cell tumor growth in in vivo by targeting the miR-509-3p/HDAC9 axis.

\section{Discussion}

Over the last decade, numerous studies have reported the aberrant expression of 1 cRNAs and their functions in NSCLC tumorigenesis and progression (28-30); thus, therapeutic strategies that target lncRNAs may hold promise for the treatment of NSCLC. Although numerous lncRNAs have been validated in the human genome (31), only a small number have been well studied in relation to NSCLC. Therefore, further investigation into the roles and relevant mechanisms of such lncRNAs is warranted. In the present study, CBR3-AS1 expression was detected in NSCLC tissues and its prognostic value was evaluated. The impacts of CBR3-AS1 on the tumorigenicity of NSCLC cell lines, and the mechanisms mediating the tumor-promoting activities of CBR3-AS1 in NSCLC progression, were also assessed.

CBR3-AS1 expression is upregulated in osteosarcoma, and this upregulation is closely associated with Enneking stage, distant metastasis and histological grade (21). High CBR3-AS1 expression has also been identified as an independent predictor of poor prognosis in patients with osteosarcoma (21). CBR3-AS1 is also highly expressed in breast cancer tissues and cell lines (32); patients with breast cancer and a high CBR3-AS1 expression level present with poorer overall and disease-free survival outcomes than those with low levels of CBR3-AS1 (32). Nevertheless, the expression profile of CBR3-AS1 in NSCLC is yet to be elucidated. In the present study, CBR3-AS1 was upregulated in NSCLC tissues and cell lines, and high levels of CBR3-AS1 were associated with a larger tumor size, advanced TNM stage and an increased incidence of lymph node metastasis. The overall survival times of NSCLC patients with high CBR3-AS1 expression were shorter than those of patients with low CBR3-AS1 expression.
These observations suggest that the high CBR3-AS1 expression observed in NSCLC is associated with poor postoperative prognosis in patients with this malignancy. Thus, CBR3-AS1 may be developed as an effective target for NSCLC diagnosis and prognosis.

Functionally, silencing CBR3-AS1 attenuates cellular proliferation, migration and invasiveness, and promotes apoptosis in osteosarcoma (21). In breast cancer, CBR3-AS1 exerts an oncogenic role by regulating cell proliferation, colony formation and apoptosis in vitro, and tumor growth in vivo (32). To better comprehend the detailed function(s) of CBR3-AS1 in NSCLC, the impacts of CBR3-AS1-knockdown on NSCLC cells were determined using a series of functional experiments in vitro and in vivo. CBR3-AS1-knockdown resulted in an obvious decrease in cellular proliferation, migration and invasiveness, as well as an increase in apoptosis in vitro. Moreover, a tumor xenograft model indicated that CBR3-AS1 inhibition hindered the tumorigenicity of NSCLC cells in vivo. These results collectively suggest that CBR3-AS1 is a potential target for NSCLC anticancer therapy.

The functions of lncRNAs are largely determined by their subcellular localization (33). To elucidate the functions of CBR3-AS1 in NSCLC, an IncRNA subcellular localization predictor (lncLocator) and nuclear-cytoplasmic fractionation were applied to identify the localization of CBR3-AS1. The results confirmed CBR3-AS1 as a cytoplasmic lncRNA and suggested that CBR3-AS1 functions as a ceRNA and sponges miRNA, thereby liberating miRNAs from their binding sites on target mRNAs. Clarification of the miRNA/mRNA axis will advance our understanding of the mechanisms by which CBR3-AS1 promotes the oncogenic potential of NSCLC cells.

Bioinformatics analysis was performed to predict the miRNAs that interact with CBR3-AS1. Among the candidates identified, miR-509-3p was selected for further experimental verification given its crucial roles in tumorigenesis and cancer progression (24-27). Luciferase reporter and RIP assays confirmed miR-509-3p as a target of CBR3-AS1 in NSCLC cells. In addition, miR-509-3p was weakly expressed and inversely correlated with CBR3-AS1 expression in NSCLC tissues. Furthermore, miR-509-3p expression was increased in NSCLC cells after CBR3-AS1 silencing. Mechanistic investigations identified HDAC9 as a direct target of miR-509-3p in NSCLC cells; HDAC9 expression was also positively regulated by CBR3-AS1, and these regulatory actions were exerted through miR-509-3p sponging. These results validate that a ceRNA regulatory pathway involving CBR3-AS1, miR-509-3p and HDAC9 exists in NSCLC cells.

HDAC9, a member of the histone deacetylase family, was discovered to play crucial roles in a number of malignant characteristics of cancer progression. HDAC9 is highly expressed in NSCLC and is associated with adverse clinicopathological characteristics and shorter overall patient survival time (34). Functionally, HDAC9 exerts oncogenic activities in NSCLC cells by promoting proliferation, colony formation, migration and invasiveness, and by inducing apoptosis. In the present study, rescue experiments demonstrated that increasing the output of the miR-509-3p/HDAC9 axis counteracted CBR3-AS1 depletion-induced inhibitory impacts on NSCLC cells. Jointly, CBR3-AS1, miR-509-3p 
and HDAC9 constitute an interactive regulatory network that exerts tumor-promoting effects in NSCLC tumorigenesis and progression. Therefore, the CBR3-AS1/miR-509-3p/HDAC9 pathway may be an effective target for the improved control of NSCLC.

The present study has two limitations. Firstly, CBR3-AS1 and miR-509-3p expression data in NSCLC from The Cancer Genome Atlas (TCGA) database were not analyzed. Secondly, the correlation between CBR3-AS1 and miR-509-3p expression in NSCLC was not examined using data from TCGA database, limitations that will be addressed in the near future. However, to the best of our knowledge, the present study is the first to highlight the cancer-promoting effects of CBR3-AS1 in NSCLC cells, both in vitro and in vivo. Mechanistically, CBR3-AS1 was found to function as a ceRNA that sponges miR-509-3p, thereby increasing HDAC9 expression. These findings may positively impact the development of novel targeted drugs and the enrichment of therapeutic strategies for NSCLC.

\section{Acknowledgements}

Not applicable.

\section{Funding}

No funding was received.

\section{Availability of data and materials}

The datasets used and/or analysed during the current study are available from the corresponding author on reasonable request.

\section{Authors' contributions}

YG and LC provided substantial contributions to the conception and design of the study. YC and JY performed flow cytometry, Transwell migration and invasion assays, tumor xenograft model construction, and RNA immunoprecipitation. All statistical analysis was executed by LC. YG and LC drafted and critically revised the manuscript for important intellectual content. All authors read and approved the final draft.

\section{Ethics approval and informed consent}

The present study was approved by the Human Ethics Committee of Weifang People's Hospital. The study was performed in accordance with the Declaration of Helsinki, and written informed consent was obtained from all participants. Animal experimental procedures were approved by the Institutional Animal Care and Use Committee of Weifang People's Hospital.

\section{Patient consent for publication}

Not applicable.

\section{Competing interests}

The authors declare that they have no competing interests.

\section{References}

1. Bray F, Ferlay J, Soerjomataram I, Siegel RL, Torre LA and Jemal A: Global cancer statistics 2018: GLOBOCAN estimates of incidence and mortality worldwide for 36 cancers in 185 countries. CA Cancer J Clin 68: 394-424, 2018.

2. Siegel RL, Miller KD and Jemal A: Cancer statistics, 2019. CA Cancer J Clin 69: 7-34, 2019.

3. Shin JY, Yoon JK and Marwaha G: Progress in the treatment and outcomes for early-stage non-small cell lung cancer. Lung 196: 351-358, 2018.

4. Vargas AJ and Harris CC: Biomarker development in the precision medicine era: Lung cancer as a case study. Nat Rev Cancer 16: 525-537, 2016

5. Inage T, Nakajima T, Yoshino I and Yasufuku K: Early lung cancer detection. Clin Chest Med 39: 45-55, 2018.

6. Hirsch FR, Scagliotti GV, Mulshine JL, Kwon R, Curran WJ Jr, Wu YL and Paz-Ares L: Lung cancer: Current therapies and new targeted treatments. Lancet 389: 299-311, 2017.

7. Ponting CP, Oliver PL and Reik W: Evolution and functions of long noncoding RNAs. Cell 136: 629-641, 2009.

8. Chen J, Wang R, Zhang K and Chen LB: Long non-coding RNAs in non-small cell lung cancer as biomarkers and therapeutic targets. J Cell Mol Med 18: 2425-2436, 2014.

9. Bian C, Yuan L and Gai H: A long non-coding RNA LINC01288 facilitates non-small cell lung cancer progression through stabilizing IL-6 mRNA. Biochem Biophys Res Commun 514: 443-449, 2019.

10. Wang Y, Luo X, Liu Y, Han G and Sun D: Long noncoding RNA RMRP promotes proliferation and invasion via targeting miR-1-3p in non-small-cell lung cancer. J Cell Biochem 120: 15170-15181, 2019.

11. Tang H, Han X, Li M, Li T and Hao Y: Linc00221 modulates cisplatin resistance in non-small-cell lung cancer via sponging miR-519a. Biochimie 162: 134-143, 2019.

12. Zhang L, Hu J, Li J, Yang Q, Hao M and Bu L: Long noncoding RNA LINC-PINT inhibits non-small cell lung cancer progression through sponging miR-218-5p/PDCD4. Artif Cells Nanomed Biotechnol 47: 1595-1602, 2019.

13. Hu X, Duan L, Liu H and Zhang L: Long noncoding RNA LINC01296 induces non-small cell lung cancer growth and progression through sponging miR-5095. Am J Transl Res 11: 895-903, 2019.

14. Solé $\mathrm{C}$ and Lawrie $\mathrm{CH}$ : MicroRNAs and metastasis. Cancers (Basel) 12: 96, 2019.

15. Deng H, Xie C, Ye Y and Du Z: MicroRNA-1296 expression is associated with prognosis and inhibits cell proliferation and invasion by Wnt signaling in non-small cell lung cancer. Oncol Lett 19: 623-630, 2020.

16. Zhou X, Liu S, Liu J, Zhang Z, Mao X and Zhou H: MicroRNA-130a enhances the killing ability of natural killer cells against non-small cell lung cancer cells by targeting signal transducers and activators of transcription 3. Biochem Biophys Res Commun 523: 481-486, 2019.

17. Yan L, Zhang Y, Li K, Wang M, Li J, Qi Z, Wu J, Wang Z, Ling L, Liu H, et al: miR-593-5p inhibit cell proliferation by targeting PLK1 in non small cell lung cancer cells. Pathol Res Pract 216: 152786, 2020.

18. Qu CX, Shi XC, Zai LQ, Bi H and Yang Q: LncRNA CASC19 promotes the proliferation, migration and invasion of non-small cell lung carcinoma via regulating miRNA-130b-3p. Eur Rev Med Pharmacol Sci 23 (Suppl 3): S247-S255, 2019.

19. Wang X, Yin H, Zhang L, Zheng D, Yang Y, Zhang J, Jiang $H$, Ling X, Xin Y, Liang H, et al: The construction and analysis of the aberrant lncRNA-miRNA-mRNA network in non-small cell lung cancer. J Thorac Dis 11: 1772-1778, 2019.

20. Li H, Guo X,Li Q, Ran P, Xiang X, Yuan Y, Dong T, Zhu B, Wang L, Li F, et al: Long non-coding RNA 1308 promotes cell invasion by regulating the miR-124/ADAM 15 axis in non-small-cell lung cancer cells. Cancer Manag Res 10: 6599-6609, 2018.

21. Zhang Y, Meng W and Cui H: LncRNA CBR3-AS1 predicts unfavorable prognosis and promotes tumorigenesis in osteosarcoma. Biomed Pharmacother 102: 169-174, 2018.

22. Livak KJ and Schmittgen TD: Analysis of relative gene expression data using real-time quantitative PCR and the 2(-Delta Delta C(T)) method. Methods 25: 402-408, 2001.

23. Wang L, Cho KB, Li Y, Tao G, Xie Z and Guo B: Long noncoding RNA (lncRNA)-mediated competing endogenous RNA networks provide novel potential biomarkers and therapeutic targets for colorectal cancer. Int J Mol Sci 20: 5758, 2019. 
24. Patil SL, Palat A, Pan Y, Rajapakshe K, Mirchandani R, Bondesson M, Yustein JT, Coarfa $\mathrm{C}$ and Gunaratne PH: MicroRNA-509-3p inhibits cellular migration, invasion, and proliferation, and sensitizes osteosarcoma to cisplatin. Sci Rep 9: 19089,2019

25. Niu L, Ni H, Hou Y, Du Q and Li H: miR-509-3p enhances platinum drug sensitivity in ovarian cancer. Gene 686: 63-67, 2019.

26. Chen W, Du J, Li X, Su J, Huang Y, Ding N, Zhang M and Jiang S: miR-509-3p promotes cisplatin-induced apoptosis in ovarian cancer cells through the regulation of anti-apoptotic genes. Pharmacogenomics 18: 1671-1682, 2017.

27. Sun J, Li J, Zhang W, Zhang J, Sun S, Li G, Song H and Wan D: MicroRNA-509-3p inhibits cancer cell proliferation and migration via upregulation of XIAP in gastric cancer cells. Oncol Res 25: 455-461, 2017.

28. Tang L, Wang T, Zhang Y, Zhang J, Zhao H, Wang H, Wu Y and Liu K: Long non-coding RNA AWPPH promotes postoperative distant recurrence in resected non-small cell lung cancer by upregulating transforming growth factor beta 1 (TGF- $\beta 1$ ). Med Sci Monit 25: 2535-2541, 2019.

29. Bai Y, Zhang G, Chu H, Li P and Li J: The positive feedback loop of IncRNA DANCR/miR-138/Sox 4 facilitates malignancy in non-small cell lung cancer. Am J Cancer Res 9: 270-284, 2019.
30. Wang D and Hu Y: Long non-coding RNA PVT1 competitively binds microRNA-424-5p to regulate CARM1 in radiosensitivity of non-small-cell lung cancer. Mol Ther Nucleic Acids 16: 130-140, 2019.

31. Kopp F and Mendell JT: Functional classification and experimental dissection of long noncoding RNAs. Cell 172: 393-407, 2018.

32. Xu L, Zhu H, Gao F, Tang Y, Zhu Y, Sun Z and Wang J: Upregulation of the long non-coding RNA CBR3-AS1 predicts tumor prognosis and contributes to breast cancer progression. Gene X 2: 100014, 2019.

33. Ogunwobi OO and Kumar A: Chemoresistance mediated by ceRNA networks associated with the PVT1 locus. Front Oncol 9: 834, 2019.

34. Ma Z, Liu D, Di S, Zhang Z, Li W, Zhang J, Xu L, Guo K, Zhu Y, Li X, et al: Histone deacetylase 9 downregulation decreases tumor growth and promotes apoptosis in non-small cell lung cancer after melatonin treatment. J Pineal Res 67: e12587, 2019. Attribution-NonCommercial-NoDerivatives 4.0 International (CC BY-NC-ND 4.0) License. 